\title{
Action Recognition for Support of Adaptive Gameplay: A Case Study of a First Person Shooter
}

\author{
S. Kazmi and I. J. Palmer \\ Department of Electronic Imaging and Media Communications, School of Informatics, University of Bradford, \\ West Yorkshire, BD7 1DP, UK \\ Correspondence should be addressed to S. Kazmi, sayedkazmi@hotmail.com
}

Received 28 July 2009; Accepted 16 January 2010

Academic Editor: Abdennour El Rhalibi

Copyright ( $\odot 2010$ S. Kazmi and I. J. Palmer. This is an open access article distributed under the Creative Commons Attribution License, which permits unrestricted use, distribution, and reproduction in any medium, provided the original work is properly cited.

\begin{abstract}
With games continuously and rapidly evolving to become more complex and sophisticated in their nature and implementation. There is a fundamental need to sustain and deliver a similarly advanced, realistic, and engaging experience for the player. The implementation of "emergence" within games as providing an effective means to sustain this engagement in conjunction with some form of action recognition mechanism for its support. More recently, games have made much of the "adaptive" mechanisms that tailor the player experience during the game, but much of this appears to be implemented by merely making the game harder according to the success of the player. Some go further than this by incorporating adaptive AI that change agent tactics to suit the player's style of play. Whilst these are clearly advances in the approach to providing a player-centric experience to engage the player, the basis and transferability of these approaches is open to question. Here we propose a limited flavour of "emergence" which can be used to support an adaptive game mechanism and so present players with different gameplay experiences based on their actions within the game.
\end{abstract}

\section{Introduction}

Computer games have an extensive history that dates back to the early fifties [1], with games continuously and rapidly evolving to become more complex and sophisticated in their nature and implementation. It is this highly advanced nature inherent in contemporary gaming alongside game platforms' current technological prowess that creates a fundamental need to sustain and deliver an advanced, realistic, and engaging experience for the player.

The implementation of "emergence" within games has been discussed $[2,3]$ as providing a possible effective means to sustain this engagement in conjunction with some form of action recognition mechanism for its support. More recently, games such as Splinter Cell, Pandora Tomorrow [4], and Lego Star Wars II [5] have made much of the "adaptive" mechanisms that tailor the player experience during the game, but much of this appears to be implemented by merely making the game harder according to the success of the player. Pro Evolution Soccer 2008 [6] goes further than this by incorporating an adaptive AI called "Teamvision" that changes the team's tactics to suit the player's style of play. Whilst these are clearly advances in the approach to providing a player-centric experience to engage the player, the basis and transferability of these approaches is open to question. Here we propose a limited flavour of "emergence" which can be used to support an adaptive game mechanism and so present players with different gameplay experiences based on their actions within the game.

\section{Aims of the Research}

The aim of the research was to investigate whether a system could be developed that continuously monitors player progression through a level, recognises particular actions made by the player, and from this information correctly predicts the level of player ability. It is proposed that this could be achieved through the development of a series of component action recognition operations to categorise the player and therefore allow the gameplay to be adapted to suit the player. The collection of component action recognition operations will be referred to as the "action recognition algorithm". 


\section{Problem Identification}

Video gameplay has often included disappointing, unrealistic and ineffective nonplayer characters (NPCs) and this is commonplace in even the most modern and highly regarded of game titles [7-9]. This view has been further validated by discussions with focus groups drawn from over ninety first, second and third year undergraduate students aged between 18 and 30, studying computer game development. The adoption of a technique that attempted to prevent monotonous gameplay and enhance the challenge presented to the player would promise to make videogames more entertaining and enjoyable. This should also offer players of differing abilities and experience an equally enjoyable game.

\section{Current Problems Providing Justification of Research}

There is little doubt that the variety and sheer number of games available offer today's game player with a wealth of options to satisfy their desire for interactive entertainment. This does not however, mean that there is no room for improvement in the experience that is provided in a given game environment, and it is possible to identify a number of areas for study that may allow the use of an in-game mechanism to tailor the user experience to their abilities.

\subsection{Difficulty}

"Part of the problem, is that many game makers are stuck with the one-size-fits-all mindset that takes little account of the different amounts of time that people have to devote to a game or for their varying skill levels" Guillemot in Ward [10].

Games often allow the user to set the difficulty level to one of a small number of possibilities, most commonly being: easy, intermediate, and hard. However, this is of little consolation to users that may find themselves belonging to an expertise level that ranges somewhere between these settings, resulting in a level that is too difficult or too easy to play. It is also hard for a new player to predict accurately and reliably which category they fall into and hence there is a risk of the player being frustrated by either too easy or too difficult gameplay. To compound this problem, many games only allow this choice to be made at the very start of the game. This can mean that if the player finds himself unable to continue through the game due to the level of skill required being beyond him, he has no other option but to restart the game at the lower difficulty setting.

\subsection{Complexity}

"An over complicated design seems to be very common in the games market, complexity in a game is not necessarily a good thing" [11].

Current games use highly complex sets of levels or stages (or variations on levels) that could effectively be replaced with a single level that adapts to the particular style and expertise of the individual player for a much more satisfying tailored experience. This makes it possible to reduce the cognitive complexity and associated learning curve that the user is subjected to in playing a multiple stage game, where each stage contains some new predefined feature.

\subsection{Lack of Realistic Intelligence in Games}

\begin{abstract}
"The original bots in FPS's were completely oblivious to their environment and used fixed scripts to attack the human player. Current bots, such as those found in Unreal are beginning to approximate the game play of humans. What if anything are they missing? Although they can react to different situations, as of yet they cannot anticipate or adapt to the behaviour of other players" Laird [12].
\end{abstract}

NPCs in current games are provided with the most basic of operational behaviour that restricts their capabilities and navigation. For example, an action instigated by the user can be misinterpreted, or even missed completely by NPCs if it falls outside the scope of their simple programmed logic. The use of adaptive gameplay would enable interaction with the user at a higher level, by predicting the intentions of the user based on previously recognised sequences of actions, rather than simply responding to low-level actions.

\subsection{Lack of Engagement}

\section{"Players expect total interactivity, they want every character to acknowledge them and react to them" Butcher and Griesemer [13].}

In their most basic guise, many FPS games simply require the player to attack and kill the "infinite" numbers of unintelligent NPCs that they encounter by using little else than primary weapon fire. During discussions with focus groups (as defined in Section 3), this has been found to directly contribute to making the player feel isolated and separate from the game, effectively and substantially eliminating the enjoyment factor that may have otherwise existed beyond the visceral "thrill of the kill" [14].

The inclusion of adaptive mechanisms within the gameplay in itself, could dramatically improve the level of player engagement by changing the gameplay to suit the style of the player and present further challenges as the player progresses, therefore providing a more convincing, interesting, and variable environment.

\subsection{Restrictive Geometry.}

"Red Faction is a new generation of first person shooters. The game has huge potential thanking Geo-Mod technology (real-time, arbitrary geometry modification) that drastically enhances realism and diversity of gameplay" Tsai [15]. 
Modern gaming environments often confine the player to travel along clearly marked, programmed routes, meaning the player only has a limited number of ways in which they can proceed from location A to location B [16]. Embedded mechanisms can support innovative features such as geometric modification, enabling the players' actions to dynamically alter their environment to suit their style of play or abilities as they progress through the game.

4.6. Stagnation. Rollings and Adams illustrate the problem of stagnation where the player advances to a particular point in the level and can proceed no further [14]; a good example of this is the unnatural progression demonstrated by "Metal Gear Solid" [17], where the player is required to physically change controller ports in order to continue with the game, the player must therefore consult the game "walkthrough" or some other third party information source to complete the game. Whilst this is an innovative game mechanism, it can be seen as counterintuitive and certainly breaks the engagement with the virtual game environment and the suspension of disbelief.

Action recognition and adaptive gameplay can remedy this by allowing for supporting multiple ways of achieving the same goal, with the method most appropriate to the players "style" as indicated by their previous actions in the game. A simple example of this could be if that the player became stuck at an early stage of the game for a period of time, because they cannot determine how to unlock a door, the game would recognise their inexperience, remove the need for a key, and unlock the door for them before they reach it or make the key more obvious and accessible.

\subsection{Predictability}

"We ended up with a game that I didn't know how to win. I didn't know which were the best strategies or tactics, even though I designed all the game's systems. That is what makes a good strategy game" Gollop in Rollings and Adams [14].

Currently available games offer only a finite set of solutions and once these have been discovered and implemented, the challenge of the game becomes obsolete, as it no longer engages the user, nor provides any further motivation for play.

Action recognition in conjunction with adaptive gameplay would ensure that there are multiple solutions to the game that become available according to the player's actions and therefore, would keep the user immersed as they look for new and novel ways of completing the game. This would be achieved since as the player's skill develops, the system recognises this, and changes the game environment to continue to offer new challenging experience.

\subsection{Lack of Reward}

"Players also expect a significant challenge, gamers have become sophisticated, many of them have played games in your genre for years and they want a challenge" Butcher and Griesemer [13].
If the player completes a series of simple tasks to achieve some subgoal within the game, they may not bother completing the other subtasks, as they do not offer any significant reward for achievement. By using adaptive gameplay, we can greatly enhance this factor as the player is required to "think for themselves", and any success is directly related to their own particular actions. This consequently increases the "feel good" and "self earned win" factors of gameplay, and can strengthen their immersion within it.

\section{Design and Initial Research}

Before considering any adaptive mechanisms that can be introduced to the gameplay, we need to provide a mechanism embedded in the system that will allow the user's actions to be monitored and recognised. This mechanism is the focus of the next section of the paper.

5.1. Approaches to Action Recognition. The basic premise of this research is that the recognition of specific actions within gameplay will enable us to ultimately gauge the level of individual player skill and style of play. However, we must first understand what the term 'action recognition' actually means and the forms that it can take.

Intille and Bobick [18] and Shafie [19] in their works regarding multiagent virtual environments, insist that action recognition is the term used for the identification of any discernible motion or behaviour of any visible character. Nigro et al. [20] refer to the term situation/manoeuvre recognition to be synonymous with action recognition and describe it as the detection and characterisation of a particular manoeuvre being undertaken by a character or object. Grimbergen and Matsubara [21] utilise yet another term; "pattern recognition", meaning to recognise the formation of objects and to define their state of play by continuously monitoring their movement.

Laird [12] has stated that in order to accurately recognise particular actions of the player, we can rely on simple Finite State Machine (FSM) based recognition mechanisms that solely concentrate on actions that the player executes through interaction with the computer. An example of such could be setting an ambush, when an NPC detects the enemy picking up a health top-up that is known to be positioned in a particular area-a set of behavioural rules can then be ready for use that best caters for a counteroffensive within that particular location.

This rigid form of action recognition, rather than alternatives that employ AI techniques such as artificial neural networks, genetic algorithms, and Bayesian networks, is preferred in many cases due to the reduced uncertainty and time involved in operation, coupled with an enhanced tuning and development capability.

From these works the authors feel that action recognition in the context of this article will conform to the characterisation made by Laird [12], as it is essentially the same as the Grimbergen and Matsubara [21], Nigro et al. [20], and Intille and Bobick [18] classifications, but offers a more precise, comprehensive, and most importantly, more fitting 
understanding of the term. Furthermore, as this technique offers a faster mode of action recognition in contrast to the alternatives mentioned, it would also be better suited to the real time environment that computer games present.

Before any development work on the action recognition algorithm can be undertaken, data regarding the different styles of game play must first be obtained to develop a reference for the proposed system. To do this, a level was designed and constructed as a test platform, in order to acquire data regarding real player activities. Here this was implemented through the use of the "Unreal Editor" [22] software.

The action recognition component of the game uses two related forms. Firstly customised triggers are positioned around the map at key strategic locations. Secondly, the interaction with game elements such as doors, access hatches, and weapons are recorded. It is also possible to log key events such as player deaths that can add to the total information either during or post any game.

The code for each trigger and door possesses an event handler, that is, activated upon the player coming within some predetermined proximity of the former, or upon the player contacting the surface of the latter. This event handler has been customised by the authors to output a string to a text file signifying its activation accordingly, therefore indicating a specific action and creating a unique series of discrete events triggered by the player.

\section{Initial Experiment}

6.1. Overview. After the level had been developed to a correctly functioning and playable standard through extensive open play-testing amongst a class of 30 unpaid, mixed sex, and ability game design students and various online users to identify any faults, it was then given to a random populace of 16 players in order to acquire data regarding the actual characteristics that belong to different categories of player for alpha testing.

6.2. Subjects. The test subjects were 16 unpaid mixed sex evaluators from a random population between the ages of 18 to 24 , ranging from complete novices to expert gamers that possessed extensive experience of first person shooter games, predominantly the same type as Unreal Tournament [22]. Each evaluation used the same machine and software in order to provide as reliable, robust, and as uniform as possible a set of observations to compare and contrast player performance against.

6.3. Task Implemented. Each evaluator was introduced to the game and its main features and controls, they were then allowed to enter the first two rooms of the level in "invincible mode." This was so that they became comfortable with the game without dying prematurely, whilst simultaneously ensuring they remained unaware of tactical NPC positioning in the later parts of the game, maintaining the element of discovery and surprise, and preserving the game's basic playability.
When each evaluator had become comfortable, the game was reset, and the player instructed to complete as much of the level as possible, within a maximum of three attempts. After undertaking their task, each evaluator was asked to complete a feedback questionnaire that was utilised to gauge their personal experience.

6.4. Player Experience. From the evaluations only five players from the entire sample population successfully completed the game within the three attempts. The level of player expertise comprised eight expert gamers, six intermediate gamers, and only two amateur players. It is evident that only those with relatively high experience from the sample population actually completed the game or made significant progress.

6.5. Data Collected. From observations made of the five best and five worst players, a number of key areas revealed distinct patterns regarding the manner in which players completed the game, each area has therefore been categorised and any important information obtained with respect to player navigation and progress has been concisely detailed within Tables 1 and 2.

6.6. Player Navigation. Five players chose an identical and completely linear route through the game (please refer to Figure 1), while a further six took the same route with negligible modification to complete the game. Therefore, in short, eleven players took a basically linear path through the game. Only three players used the safe passage, whilst only one player used the rapid access to Level 2. Of the five players that progressed to the second floor "fish bone" corridor, three utilised the passing place segments.

It is clear that players mainly follow the most apparent path through the game facing the least possible resistance, whilst being oblivious to even the most apparent shortcuts. Recognition of this behaviour should support prediction of player behaviour and allow the development of navigational algorithms for NPC counter responses in accordance with them.

6.7. Player Deaths. Room Four had the highest player death rate; the worst four players were killed in this room, as a direct result of amateur players' first contact with enemy NPCs and struggling to overcome them. Room Six saw the next highest death rate, and this was attributed to amateur players targeting NPCs placed within Room Six's exit corridor, but accidentally firing upon the exit door upon its closure. This information provides an accurate and essential insight into the rated skill level of each individual player.

\section{From Events to Actions: Identifying Characteristics}

From observations of player deaths incurred, it can be seen that four fundamental actions/events can be identified, that can each be extrapolated into specific reference points 
TABLE 1: Fundamental information regarding the five best players.

\begin{tabular}{|c|c|c|c|c|c|}
\hline Subject number & 10 & 9 & 5 & 4 & 2 \\
\hline $\begin{array}{l}\text { Rooms } \\
\text { completed }\end{array}$ & 14 & 12 & 16 & 14 & 11 \\
\hline End status & Game Completed & Game Completed & Game Completed & Game Completed & Game Completed \\
\hline $\begin{array}{l}\text { Level of } \\
\text { expertise }\end{array}$ & Expert & Expert & Expert & Expert & Expert \\
\hline $\begin{array}{l}\text { Secret passage } \\
\text { used? }\end{array}$ & Yes & Yes & No & No & No \\
\hline $\begin{array}{l}\text { Quick access } \\
\text { used (shortest } \\
\text { Path) }\end{array}$ & No & No & No & No & Yes \\
\hline $\begin{array}{l}\text { Staircase access } \\
\text { used? }\end{array}$ & Yes & No & No & Yes & No \\
\hline Linear path & No & No & No & No & No \\
\hline $\begin{array}{l}\text { Linear path with } \\
\text { slight } \\
\text { modification }\end{array}$ & Yes & Yes & Yes & Yes & No \\
\hline Lives given & 1 & 1 & 1 & 3 & 1 \\
\hline $\begin{array}{l}\text { Weapon/health } \\
\text { Pickup rooms } \\
\text { utilised }\end{array}$ & No & No & Yes, 3 & Yes, 2 & No \\
\hline $\begin{array}{l}\text { Corridor } \\
\text { segments } \\
\text { utilised? }\end{array}$ & Yes & Yes & No & No & Yes \\
\hline
\end{tabular}

TABLE 2: Fundamental information regarding the five worst players.

\begin{tabular}{|c|c|c|c|c|c|}
\hline Subject number & 1 & 12 & 13 & 14 & 16 \\
\hline Rooms completed & 4 & 4 & 4 & 4 & 6 \\
\hline End status & Killed & Suicide & Killed & Killed & Suicide \\
\hline Level of expertise & Novice & Novice & Novice & Novice & Novice \\
\hline Secret passage used? & No & No & No & No & No \\
\hline $\begin{array}{l}\text { Quick access used } \\
\text { (shortest Path) }\end{array}$ & No & No & No & No & No \\
\hline Staircase access used? & No & No & No & No & No \\
\hline Linear path & No & Yes & Yes & Yes & No \\
\hline $\begin{array}{l}\text { Linear path with } \\
\text { slight modification }\end{array}$ & Yes & - & - & - & Yes \\
\hline Lives given & 1 & 2 & 2 & 1 & 1 \\
\hline $\begin{array}{l}\text { Weapon/health } \\
\text { pickup rooms utilised }\end{array}$ & No & No & No & No & No \\
\hline
\end{tabular}

to provide a good indication of amateur and experienced gameplay.

\subsection{Characteristics of Amateur Players}

(1) The first characteristic of interest for identifying amateur players is where the player is suddenly caught within the resultant blast radius of their own weapon, in relation to a stationary object such as a wall, and subsequently suffering massive health loss or death.
(2) Secondly (and closely related), is where a player unwittingly commits suicide by firing into a seemingly open corridor, which in fact, has a door momentarily being open. The closing of the door results in the same "blast radius" effect; exposing the player to the lethal effect of their own weapon.

(3) Thirdly, the player expending a great deal of ammunition with little effect on the resident NPC populace, leaving in some cases insufficient ammunition for any further combat. 


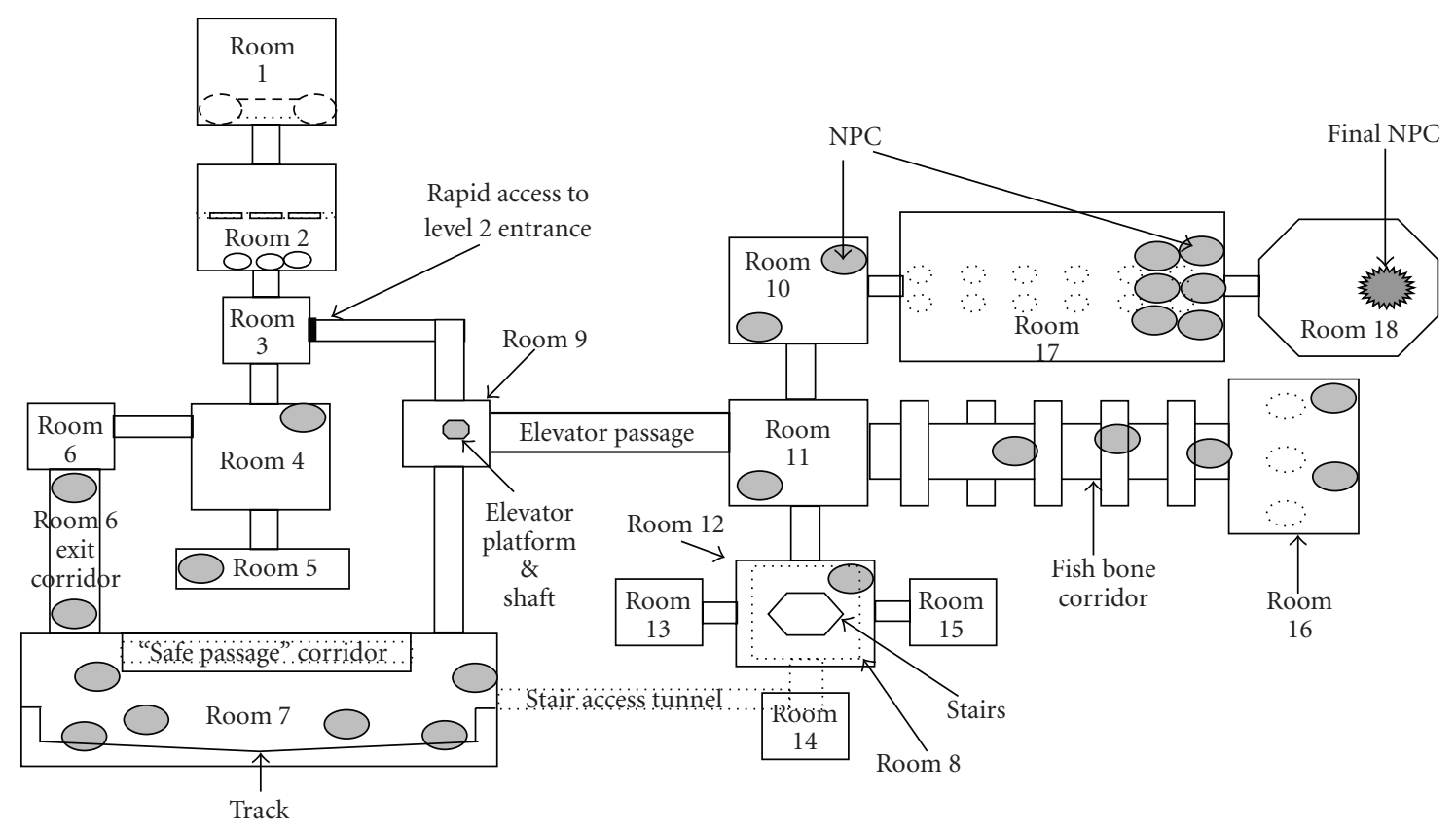

Figure 1: Level design plan.

(4) Lastly, player health diminishing significantly over a short period of time within the early stages of the game, clearly demonstrating the player possesses a poor level of gaming proficiency in avoiding damage from NPC attacks.

\subsection{Characteristics of Experienced Players}

(1) The first characteristic of experienced players is the ability to successfully defeat multiple NPCs over a short period of time, indicating the expenditure of negligible effort.

(2) Secondly, the player demonstrating use of a killzone, where they simply "stand and snipe" from some specific location to defeat multiple NPCs.

(3) Thirdly, players adopting tactics such as using passing places in corridors to block NPC fire and to launch successful strategic counterattacks from.

(3) Lastly, adopting the strategy of launching attacks solely from rooms within the game that provide a high level of safety for them with negligible possibility of harm to overcome NPC populaces, and only navigating rooms if they are perceived to be absolutely essential to complete the game.

7.3. Automating the Action Recognition Process. Once these characteristics had been determined by comparing the test results against the ability of the players, an automated recognition mechanism needed to be developed. As discussed earlier, an FSM approach was proposed, and due to the discrete nature of the events generated by the players' actions. This was simple to implement due to the support in the game engine for FSMs as an inherent mechanism for the AI of the NPCs. The experiment was then repeated with the same players after a significant length of time to reduce the effect of familiarity. No introductory session was given this time and the players were just given the level to play from the start.

The results were remarkably similar to the original experiment, expert players skill level was correctly determined by the FSM, through continually gauging player performance over the duration of play. For example, if the player was able to defeat two or more NPCs in less than 25 seconds, or is able to overcome two or more NPCs from a single map location by using "stand and snipe" killzones, these were used to suggest expert player behaviour.

7.4. Initial Findings. We have demonstrated that it is indeed possible to develop an algorithm that can identify particular player actions being instigated and allow us to correlate these actions to the expertise level of each individual player. This makes it possible to develop a strategy and a range of reference points according to which the game can be adapted to the player's specific needs and enhance the overall gaming experience provided. This recognition approach also informs our development of adaptive game mechanisms, since the actions that allow us to categorise the players offer an insight into the very aspects of the game that can be adapted to suit the player better.

The key mechanisms implemented within the level for expert rated players consisted of:

(i) A "Modify Geometry" mechanism (Figure 2) that changes the environment such that it is more difficult to navigate safely. In this particular example we dynamically modify the "fishbone" corridor (Figure 1) to restrict the degree of available player 


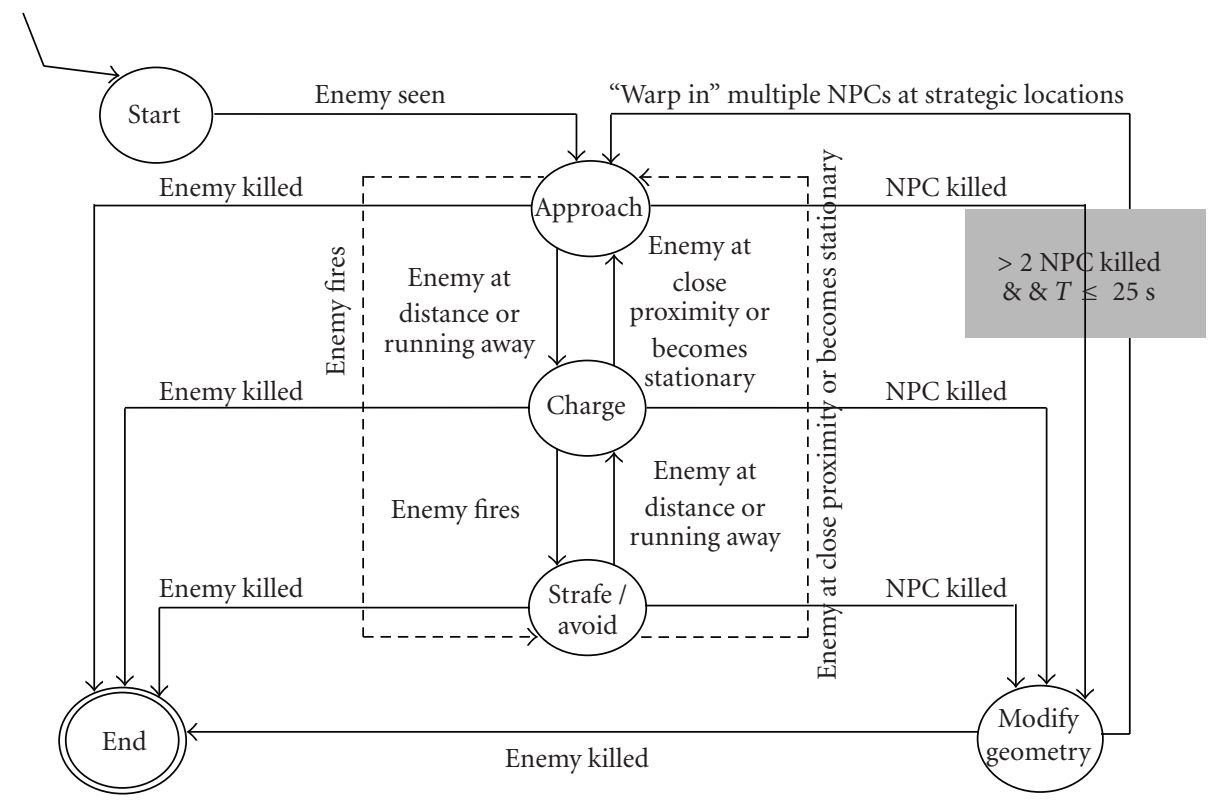

(a)

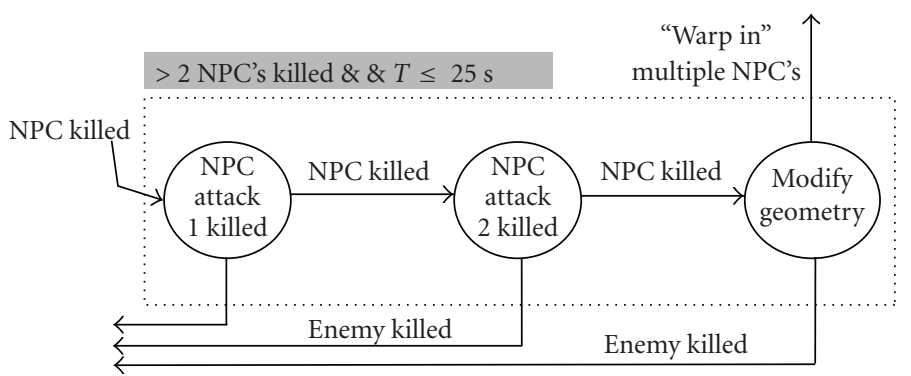

(b)

FIgUre 2: (a) Proposed additional "Passage Attack" finite state detailed. (b) "Modify Geometry" state detailed.

movement/navigation, and position a small number of NPCs at the rear to remove the hiding places for the player and increase the difficulty of this section.

(ii) A "Kill Zone Counter" mechanism (Figure 3), that enables the NPC populace to correctly recognise a situation where the player simply stands and snipes from a particular point using minimal movement to overcome NPC populaces, allowing the game to take remedial action by dispatching NPC's through an alternative route providing more of a challenge to the player.

(iii) A "Lure" mechanism (Figure 4), that operates in conjunction with the "Killzone" mechanism (Figure 3) to consequently draw the player from an area that provides a high degree of safety for them, into an area that provides sole maximum advantage for the NPC, in order to generate an ambush type scenario.

The key mechanisms implemented within the level for amateur players were as follows.

(i) An "Automatic Blast Radius Adjustment" mechanism, that after each instance of player instigated weapon fire, autonomously reduces the resultant blast radius expended to a safe degree, in relation to its proximity to the player's current location, implemented by altering a simple "Damage" logic parameter in the weapon fire class and therefore not needing an FSM.

(ii) An "Automatic Weapon Directional Control" mechanism (Figure 5), that limits player weapon activation to the direction of unobstructed NPC's alone, in order to remedy the problem of premature death by suicide, in addition to wasteful artillery expenditure.

(iii) An "Automatic NPC Skill Reduction" mechanism (Figure 6) that attempts to autonomously diminish the skill level and sensing capabilities of the NPC populace used by the game to a level in accordance with amateur player skill.

The reason for the adoption of these particular Finite State Machines is because they provide a simple and efficient means to adapt the game to provide an effective enhancement of the challenge afforded to the player.

The next stage of this work is to repeat the experiment with the new adaptive mechanisms in place to conclude, 


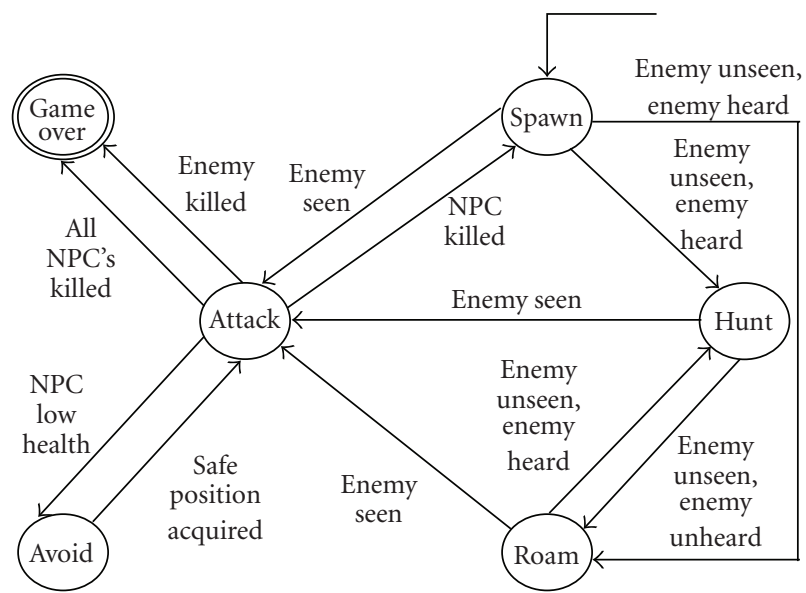

(a)

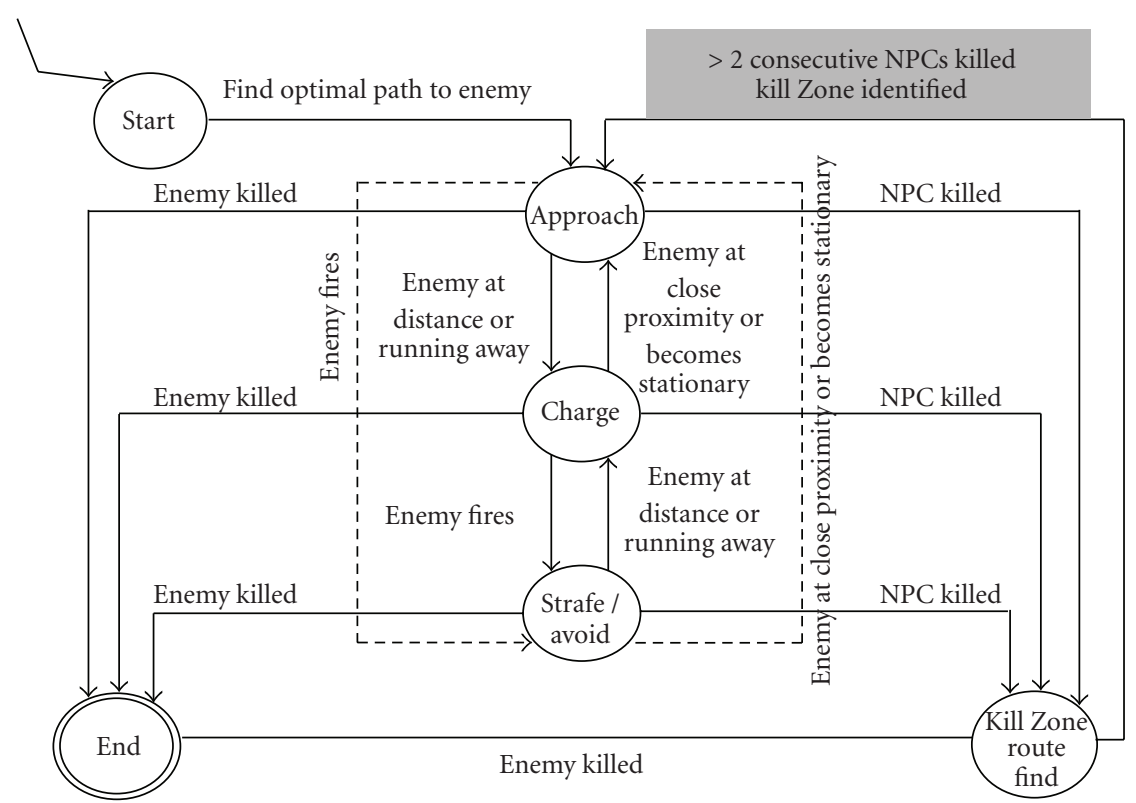

(b)

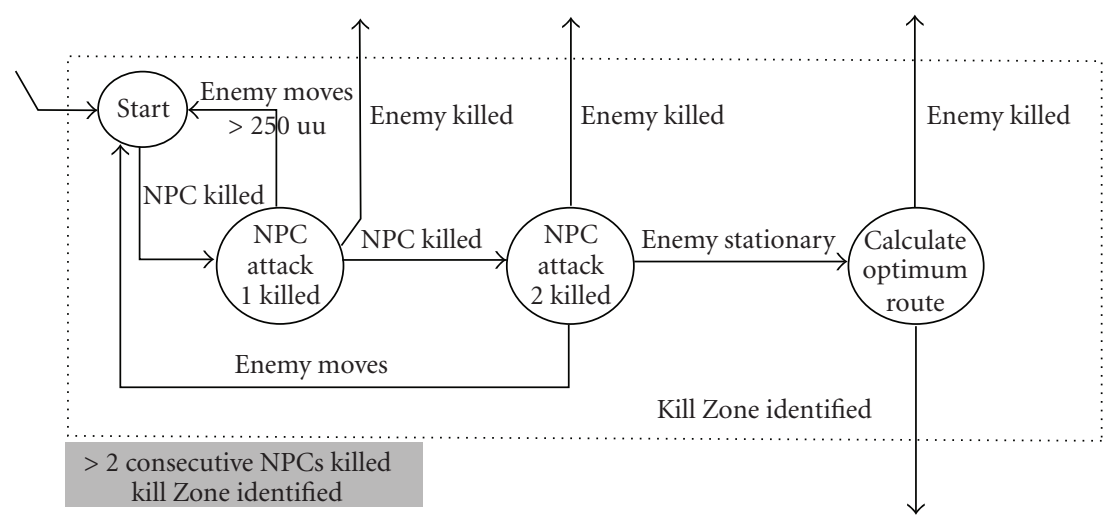

(c)

Figure 3: (a) Proposed antikill zone nonplayer character FSTN. (b) Anti-Kill zone "Attack" state detailed. (c) "Kill Zone Identified" transition detailed. 


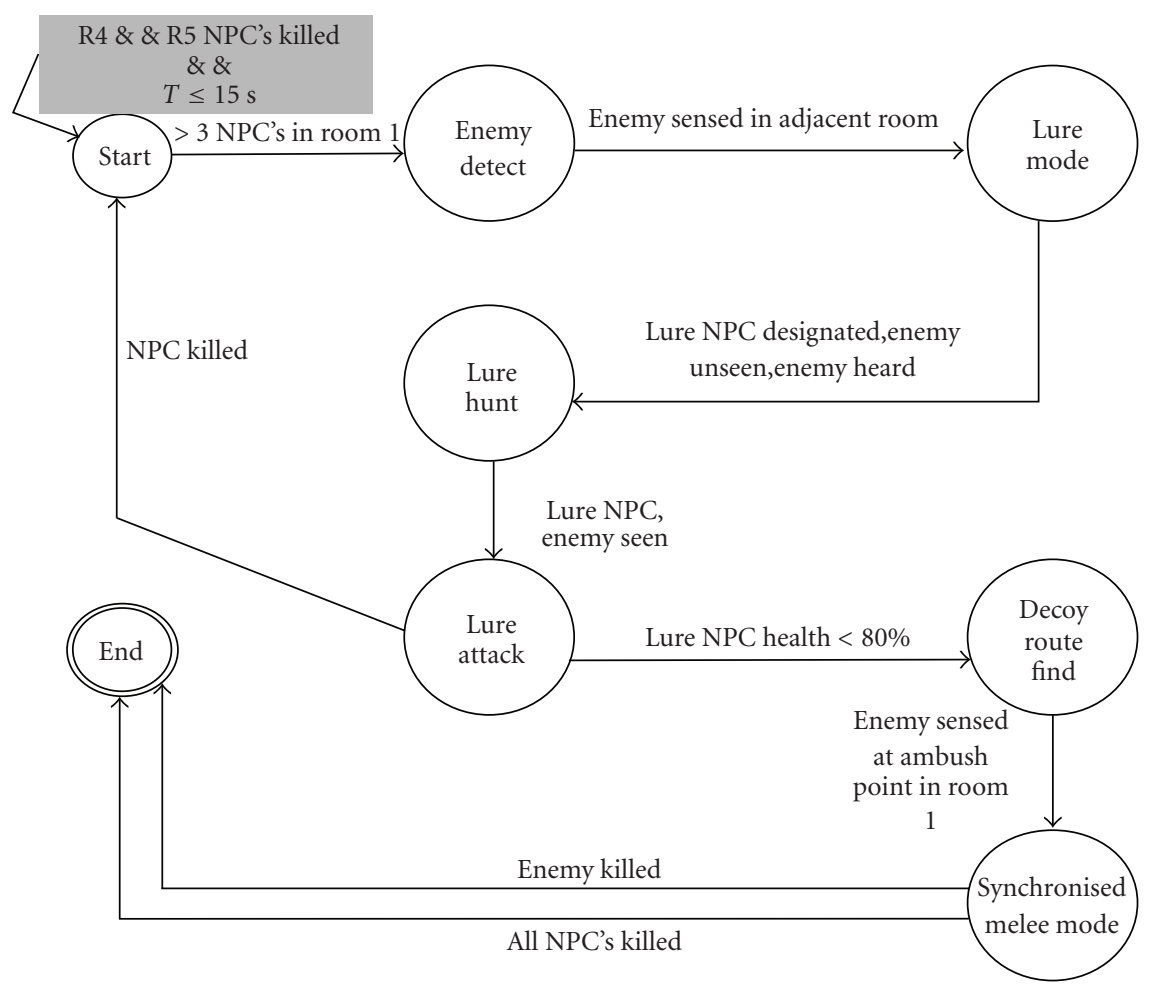

FIgURE 4: Proposed lure state transition detailed.

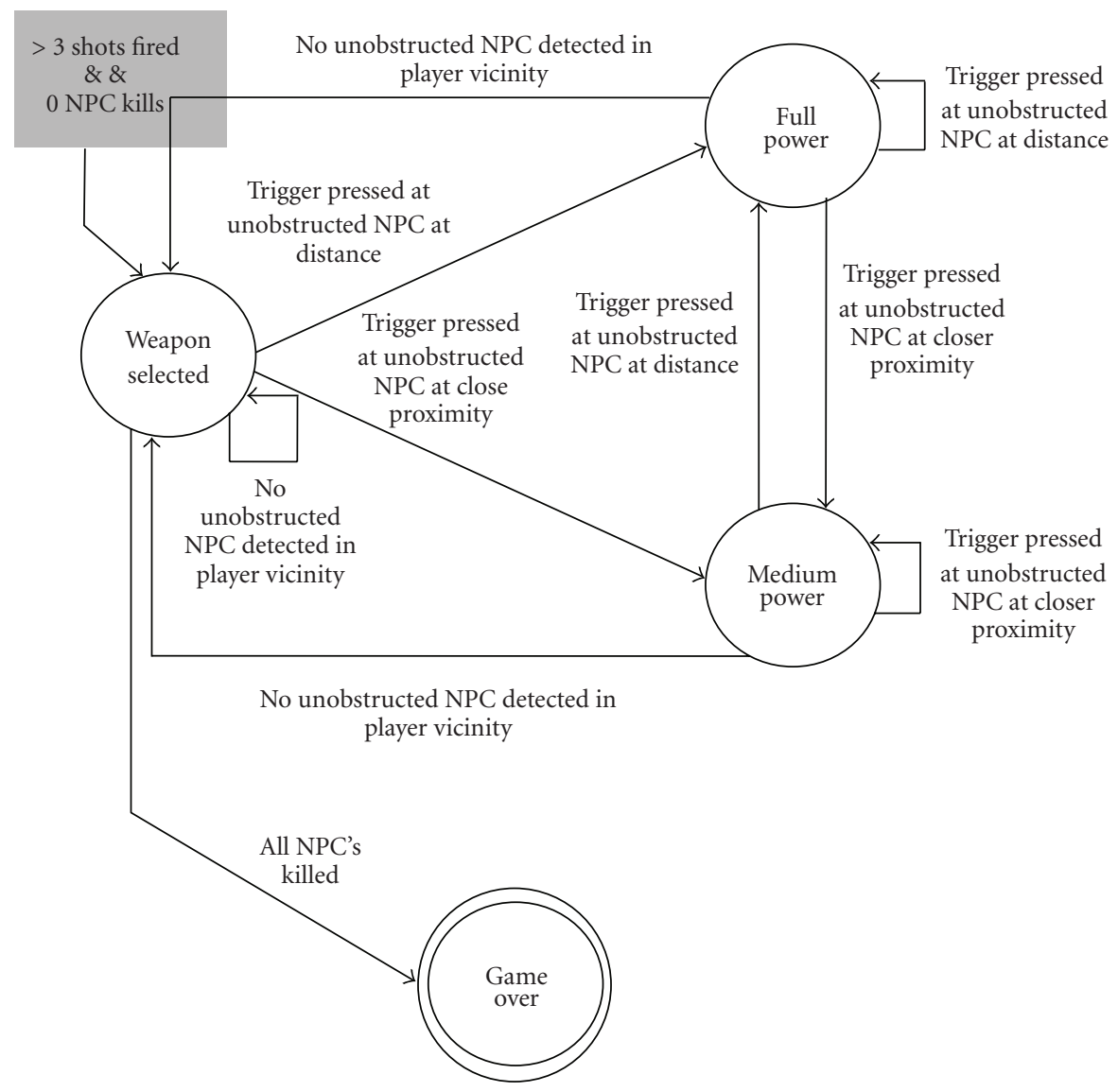

FigurE 5: Proposed autonomous weapon directional control FSA. 


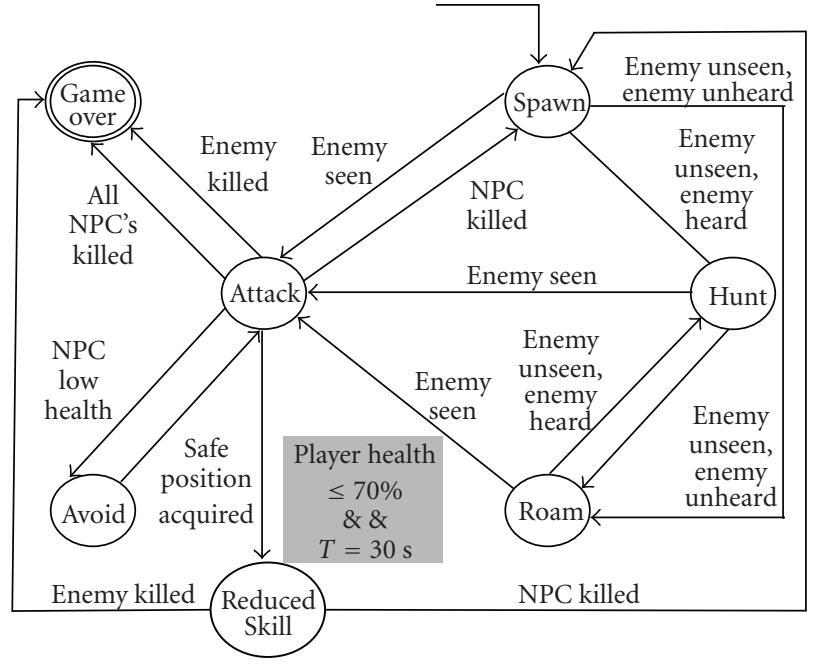

(a)

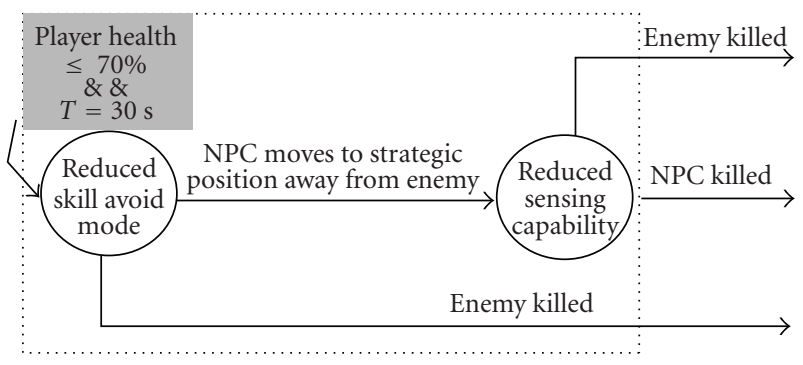

(b)

FIGURe 6: (a) Proposed reduced skill nonplayer character action finite state automaton. (b) "Reduced Skill" finite state detailed.

validate, and implement these together with the successful recognition that has already been achieved.

\section{Secondary Experiment}

In order to enable us to effectively, reliably, and robustly compare and contrast between the outcomes of the initial and consequent testing, it is essential that we adopt an identical methodology for this period of testing but with different test subjects.

This secondary testing utilised 36-test subjects, formed from two equally sized groups of unpaid, mixed sex amateur, and expert rated players.

8.1. Data Collected. Again from observations made of the five best and five worst players, a number of key areas revealed distinctive patterns regarding the manner in which players completed the game, each area has therefore been categorized and any important information obtained with respect to player performance and progress has been detailed within Tables 3 and 4.

8.2. Player Progress. As outlined earlier, the experiment undertaken used two groups, each comprising of eighteen players that were carefully categorised according to their stated level of skill, one group implementing a sole populace composed of expert players and the other amateur players, respectively.

After careful analysis of the results it has been concluded that $61 \%$ of players from the entire sample populace had successfully completed the game, in contrast to only $31 \%$ of players before the modifications detailed had been implemented, effectively doubling the player success rate, that we can attribute to the introduction of the action recognition algorithm within this test.

More importantly, the results illustrate that there was a comparable equilibrium of successful play across each of the two skill groups, as eleven players from each of the two groups successfully completed the game.

It was also discovered that 24 players $(67 \%)$ from the entire sample populace successfully negotiated twelve or more rooms, a dramatic contrast to only $25 \%$ previously. It is evident that the introduction of the action recognition algorithm has yielded a dramatic increase in the consistency of successful completion of the game for players of both skill sets.

8.3. Player Deaths Incurred. Room Seventeen possessed the highest death rate, 13 players (36\%) from the entire sample populace were killed in this room with the only other casualty occurring in room seven, in contrast to the previous experiment that saw four players $(25 \%)$ killed in room four and five players $(31 \%)$ being killed in room six.

The earlier experiment had ascertained that the majority of these deaths were due to amateur players being overwhelmed by NPCs upon initial contact and in the second instance being killed by the blast radius of accidental player weapon fire. Secondary testing has now identified that amateur player deaths have decreased sharply from $56 \%$ of players to $28 \%$.

In addition, successful recognition of novice player skill and correct autonomous adaptation of NPC behaviour to a suitable level has ensured an effective amateur player challenge corresponding to their gaming experience.

Conversely, for expert players the modifications have lead to a comparable change, that is, ultimately an increased number of player deaths (39\% of expert players, up from $6 \%$ as observed previously) through recognition of player actions and successful adaptation of NPC counter measures. Of these measures the most significant is the capacity for dynamic transformation of geometry (Figure 2) in addition to the capability of NPCs to recognise and prevail over player designated "stand and snipe" kill zones (Figure 3 ).

Therefore in short, similar to the player progress section outlined previously, the implementation of the action recognition algorithm has significantly enhanced equitable gameplay with respect to recorded fatalities belonging to both skill sets.

8.4. Action Recognition Algorithm Operational Performance. The operation of the various components that together comprise the action recognition algorithm implemented within the test map for the five best and worst players can be observed in Table 5 . 
TABLE 3: The performance of the five best players.

\begin{tabular}{|c|c|c|c|c|c|}
\hline Subject number & 1 & 2 & 3 & 4 & 8 \\
\hline Rooms completed & 12 & 12 & 12 & 12 & 14 \\
\hline End status & Completed & Completed & Completed & Completed & Completed \\
\hline Level of expertise & Expert & Expert & Expert & Expert & Expert \\
\hline Number of keys collected & 3 & 2 & 3 & 3 & 2 \\
\hline Secret passage used? & No & No & No & No & No \\
\hline Quick access used? (Shortest Path) & Yes & No & No & Yes & No \\
\hline Staircase access used? & No & No & No & No & Yes \\
\hline Linear path? & No & No & No & No & No \\
\hline Linear path with slight modification & Yes & Yes & Yes & Yes & Yes \\
\hline Lives given & 1 & 1 & 1 & 1 & 1 \\
\hline Weapon/Health pickup rooms utilised & No & No & No & No & Yes, 2 \\
\hline
\end{tabular}

TABLE 4: The performance of the five worst players.

\begin{tabular}{lccccc}
\hline Subject number & 9 & 12 & 14 & 15 \\
Rooms completed & 10 & 10 & 11 & 10 & 12 \\
End status & Killed & Killed & Killed & Killed & Killed \\
Level of expertise & Amateur & Amateur & Amateur & Amateur \\
Number of keys collected & 1 & 1 & 2 & 1 & Amateur \\
Secret passage used? & No & No & No & No & No \\
Quick access used? (Shortest Path) & No & No & No & No & No \\
Staircase access used? & No & No & Yes & No & Yes \\
Linear path? & Yes & - & 2 & - & 2 \\
Linear path with slight modification & - & 2 & No & No & No \\
Lives given & No & & No & No \\
Weapon/Health pickup rooms utilised & & &
\end{tabular}

The table shows the triggering of the component FSNs that together comprise the action recognition algorithm in relation to individual player performance.

We can safely ascertain that from the player performance logs generated that no player triggered the entire functional capability of the action recognition algorithm, whilst simultaneously it can also be safely concluded that all players were each subjected to at least a single form of game adaptation through the newly implemented mechanisms.

Expert players triggered the use of all three of the modifications designed in accordance to their skill rating, $15(83 \%)$ expert players (43\% of total player populace) activated the "kill zone" NPC counter offensive mechanism (Figure 3), $11(61 \%)$ of these same players (31\% of the total player populace) each subsequently activated the NPC "lure" modification (Figure 4), this latter group manifested itself as the successful expert player populace.

Additionally, no expert player initiated any game behavioural adaptation designed for amateur players, illustrating the efficiency of the action recognition component of the algorithm with respect to correct detection of player skill level.

However, the "modify geometry" mechanism (Figure 2), in contrast to the others implemented was not only activated for each of the eighteen (100\%) expert players $(50 \%$ of total player populace) but also for one amateur player $(2.8 \%$ of total players), therefore executing for 19 players (53\%) in total, 12 of these players (33\% of total player populace) comprising of 11 experts and 1 amateur successfully completed the map, this statistic directly contributes to it being the most utilized of all the modifications implemented. It is also evident that at that specific point within the game this amateur player had demonstrated expert player characteristics and consequently triggered an appropriate adaptive feature, and although this would make it more difficult for them to complete the map, this demonstrates the ability of the algorithm to cater effectively for player behaviours that may not inherently belong to a specific level of player expertise alone and be able to provide a more tailored and challenging game for the individual.

Conversely, of the modifications designed to specifically cater for amateur players, each of the eighteen amateur test subjects (50\% of total player populace) activated the automatic directional weapon discharge control modification (Figure 5), fifteen (83\%) amateur players ( $42 \%$ of total player populace) also had the blast radius of their weapons autonomously restricted, only nine (50\%) of these amateur test subjects ( $25 \%$ of total player populace) proved to be 
TABLE 5: Action recognition algorithm function in relation to player performance.

\begin{tabular}{|c|c|c|c|c|c|c|c|c|c|c|}
\hline \multirow[b]{3}{*}{ Modifications } & \multicolumn{5}{|c|}{ Expert players } & \multicolumn{5}{|c|}{ Amateur players } \\
\hline & \multicolumn{5}{|c|}{ Five best } & \multicolumn{5}{|c|}{ Five worst } \\
\hline & 1 & 2 & 3 & 4 & 5 & 9 & 10 & 11 & 12 & 13 \\
\hline Automatic blast radius adjustment & $\mathrm{X}$ & $\mathrm{X}$ & $\mathrm{X}$ & $\mathrm{X}$ & $\mathrm{X}$ & $\sqrt{ }$ & $\sqrt{ }$ & $\sqrt{ }$ & $\sqrt{ }$ & $\sqrt{ }$ \\
\hline Automatic weapon directional control & $\mathrm{X}$ & $\mathrm{X}$ & $\mathrm{X}$ & $\mathrm{X}$ & $\mathrm{X}$ & $\sqrt{ }$ & $\sqrt{ }$ & $\sqrt{ }$ & $\sqrt{ }$ & $\sqrt{ }$ \\
\hline Automatic NPC skill reduction & $\mathrm{X}$ & $\mathrm{X}$ & $\mathrm{X}$ & $\mathrm{X}$ & $\mathrm{X}$ & $\sqrt{ }$ & $\sqrt{ }$ & $\sqrt{ }$ & $\sqrt{ }$ & $\sqrt{ }$ \\
\hline Modify geometry & $\sqrt{ }$ & $\sqrt{ }$ & $\sqrt{ }$ & $\sqrt{ }$ & $\sqrt{ }$ & $\mathrm{X}$ & $\mathrm{X}$ & $\mathrm{X}$ & $\mathrm{X}$ & $\mathrm{X}$ \\
\hline Kill zone counter & $\sqrt{ }$ & $\sqrt{ }$ & $\sqrt{ }$ & $\sqrt{ }$ & $\sqrt{ }$ & $\mathrm{X}$ & $\mathrm{X}$ & $\mathrm{X}$ & $\mathrm{X}$ & $\mathrm{X}$ \\
\hline NPC lure & $\sqrt{ }$ & $\sqrt{ }$ & $\sqrt{ }$ & $\sqrt{ }$ & $\sqrt{ }$ & $\mathrm{X}$ & $\mathrm{X}$ & $\mathrm{X}$ & $\mathrm{X}$ & $\mathrm{X}$ \\
\hline
\end{tabular}

successful players. Furthermore, 13 (72\%) amateur players (36\% of total player populace) triggered the autonomous nonplayer

character skill reduction mechanism (Figure 6), only six $(18 \%)$ of these were successful in completing the actual game.

As acknowledged previously, the vast majority of the amateur players did not activate any mechanism designed to cater for expert rated players, and this again illustrates the correct gauging of skill by the action recognition algorithm and indeed its effective function.

We can determine that each of the amateur player modifications have been successfully triggered and had an effect on performance, although the weapon directional discharge modification (Figure 5) has proven to be the most significant for those having a novice level of skill, demonstrated by the fact that all eighteen successful amateur players triggered this.

In terms of usefulness the weapon directional control mechanism (Figure 5) was closely followed by the automatic blast radius reduction feature as it was utilised by fifteen (83\%) amateurs (42\% of total populace) with nine $(50 \%)$ of these being successful players $(25 \%$ of the total player populace).

\section{Conclusions and Future Work}

9.1. Context. As asserted earlier, the research objective is in essence an attempt to endow machine-based logic with the ability to effectively identify particular player actions and enable autonomous game adaptation to significantly increase player engagement, satisfaction, and ultimately enhance the game experience provided.

This has necessitated the development of a prototype game environment embedded with a series of adaptive component-based mechanisms that in conjunction provide a significant degree of adaptive game behaviour. This required the development of a system to assess the techniques adopted by the player in their progression throughout the level and secondarily to gauge the effectiveness of the algorithm implemented to validate the research itself.

The following provides a concise account of the authors' conclusions derived from completing the research, how they essentially correlate to the original hypothesis with respect to the key design decisions and ultimately attempts to identify any factors that signify the success of the work undertaken.

9.2. Results Acquired. The initial hypothesis was that the construction of an adaptable gaming environment provides an enhancement to the effectiveness and engagement of conventional FPS gameplay for both amateur and expert players alike.

The work described here has shown that the implementation of the algorithm developed has not only introduced a highly equitable and suitable gameplay experience for both amateur and expert players, it has also enhanced successful game completion and further increased player game progression across the player populace sampled.

Further analysis of the tests has allows us to conclude that the automatic NPC skill reduction feature (Figure 6) followed by the autonomous weapon directional control mechanism (Figure 5) are the most effective amateur gaming modifications undertaken.

For expert players the dynamic geometry modification (Figure 2), followed by the killzone counter mechanism (Figure 3), provided the most significant impact in terms of effectiveness.

9.3. Inference. The methodology employed has demonstrated that it is possible to develop an effective algorithm that can discern between particular player actions being instigated and can subsequently recognise skill levels and provide a suitably adapted player experience.

It can be concluded that the action recognition algorithm developed by the authors is able to correctly identify the skill level for players, but more importantly that it allows the introduction of a more even gameplay experience for players belonging to differing levels of proficiency in a manner that is unobtrusive. The actual design for each of the finite state automatons that characterise the core functionality of the overall algorithm have demonstrated their effectiveness in both terms of correct intended operation and reliable function.

The research has therefore shown that finite state automata provide an effective means to recognise player lowlevel gaming actions in order to enable modification of the game environment and the associated scripted behaviour 
in the game to successfully modify the gameplay. Perhaps more importantly, in attempting to determine whether the adaptable game environment provided any significant enhancement to the gameplay for players with diametrically opposed levels of gaming proficiency, the results shown here lead the authors to conclude that the answer is a positive one.

9.4. Future Directions. There still remains significant potential for further development. Currently the algorithm is composed from a variety of functionally independent mechanisms that are each executed in response to particular player initiated actions. It would be interesting to develop a further level of function that observes the activation of each of these particular component mechanisms and attempts to autonomously weight each in relation to player progression throughout the level. This would enable the continuous incremental construction of a gaming profile that observes the behaviour of each player in order to determine proficiency and ultimately modify the game for the player. Therefore if three players each triggered a particular set of component AI mechanisms and consequently completed the game, the algorithm deployed should recognise the high statistical likelihood that these players must possess a reasonable level of gaming proficiency and ultimately adapt the game so that it is more difficult for successive players that adopt a similar route through to completion. With the proliferation on online player profiles and "achievement" records, it should also be possible to transfer this profile between games within genres, or with an even higher level interpretation even between genres.

There still remain a number of further adaptive mechanisms that could each be implemented to provide a more comprehensive algorithm, such as audio announcements to warn the amateur player of impending exposure to hazards and autonomous amplification of player weapon potency. Conversely, for expert players the algorithm could also implement NPCs that each have the ability to activate doors and continuously roam the confines of the level in its entirety and perhaps additionally maintain a navigational game tree of the game space to provide optimal NPC navigation and path finding capability in contrast to the player character. Additionally, the algorithm could quite simply introduce a temporal weighting consideration spanning the entire period of play in relation to player progression, in order to enhance the efficiency and effectiveness of autonomous determination of player skill.

Although the action recognition algorithm has been designed to operate with a 3D FPS game environment, a natural avenue of investigation would be to modify the algorithm so that it provided similarly adaptive features for other game environments and genres such as role playing games, vehicle simulations, and other genres. More importantly the primary future direction that the authors envisage is that of attempting to adapt the algorithm so that instead of recognising and responding to the actions of a single player, it has the capacity to support multiple player action recognition and so offer adaptive gameplay in a multiplayer environment to balance the abilities of players and allow matches between different abilities to offer an equitable experience to all. This would enable the effective implementation of the algorithm within online games (including MMOGs) that are a rapidly expanding area within contemporary gaming, although this would necessitate a more efficient algorithm that can be more rapidly executed, stored and distributed across a network.

Recent advances in processing power, network bandwidth, together with the greatly decreasing costs of expansive memory will make this a genuine possibility within the very near future and perhaps more importantly one the authors would like to personally determine.

\section{References}

[1] H. Hirsh, M. Buro, R. Korf, M. Littman, B. Sheppard, and J. Schaeffer, "Playing with AI: trends and controversies," IEEE Intelligent Systems, vol. 14, no. 6, pp. 8-18, 1999.

[2] P. Sweetser, Emergence in Games, Charles River Media, Boston, Mass, USA, 2007.

[3] H. Smith and R. Smith, "Would the real emergent game play please stand up," in Proceedings of the Game Developers Conference (GDC '04), 2004, http://www.planetdeusex.com/ witchboy/GDC04_Emergence/sld001.htm.

[4] Ubisoft, "Splinter Cell: Pandora Tomorrow," Game for the Microsoft Xbox 360, 2007.

[5] Activision, "Lego Star Wars II: The Original Trilogy," Game for the PC, 2006.

[6] Konami, "Pro Evolution Soccer 2008," Game for the Sony Playstation 3, 2007.

[7] J. Schaeffer, V. Bulitko, and M. Buro, "Bots get smart," IEEE Spectrum, vol. 45, no. 12, pp. 48-56, 2008.

[8] A. Nareyek, "Game AI is dead. Long live game AI!", IEEE Intelligent Systems, vol. 22, no. 1, pp. 9-11, 2007.

[9] D. Fu and R. Houlette, "Putting AI in entertainment: an AI authoring tool for simulation and games," IEEE Intelligent Systems and Their Applications, vol. 17, no. 4, pp. 81-84, 2002.

[10] Y. Guillemott and M. Ward, "Computer Games Learn New Tricks,” BBC News Online, May 2008, http://news.bbc.co.uk/ 1/hi/technology/3257458.stm.

[11] A. Rollings and D. Morris, Game Architecture and Design, Coriolis Publishing, Arizona, USA, 2000.

[12] J. E. Laird, "It knows what you're going to do: adding anticipation to a Quakebot," in Proceedings of the Spring Symposium on Artificial Intelligence and Interactive Entertainment (AAAI'00), vol. 2, pp. 41-50, Palo Alto, Calif, USA, 2000.

[13] C. Butcher and J. Griesemer, "The illusion of intelligence: the integration of AI and level design in Halo," Bungie.com, December 2003, http://halo.bungie.org/misc/gdc .2002.haloai/talk.html.

[14] A. Rollings and E. Adams, Andrew Rollings and Ernest Adams on Games Design, New Riders, Indianapolis, Ind, USA, 2003.

[15] J. Tsai, “The Red Faction Interview," The Daily Telefrag, January 2004, http://articles.dailytelefrag.com/index.php?type= id\&action=print\&publ_id=48644.

[16] L. M. Van Driel, Semantic navigation in videogames, M.S. thesis, Delft University of Technology, Delft, The Netherlands, 2008.

[17] Konami, "Metal Gear Solid," Game for the Sony Playstation 2, 1998.

[18] S. S. Intille and A. F. Bobick, "Framework for recognizing multi-agent action from visual evidence," in Proceedings of the 16th National Conference on Artificial Intelligence (AAAI '99), pp. 518-525, Orlando, Fla, USA, 1999. 
[19] M. A. L. Shafie, Action recognition and motion planning for synthetic actors in virtual environments (VES): behavioural animation techniques for synthetic actors in VEs: action formalisation within the framework of path planning for several actors, Ph.D. thesis, The University of Bradford, Bradford, England, 2001.

[20] J.-M. Nigro, S. Loriette-Rougegrez, and M. Rombaut, "Driving situation recognition with uncertainty management and rulebased systems," Engineering Applications of Artificial Intelligence, vol. 15, no. 3-4, pp. 217-228, 2002.

[21] R. Grimbergen and H. Matsubara, "Pattern recognition for candidate generation in the game of shogi," in Games in AI Research, H. J . van den Herik and H. Iida, Eds., pp. 97-108, Van Spijk, Venlo, The Netherlands, 2000.

[22] Epic, “Unreal Tournament 2003," PC video game, 2003. 

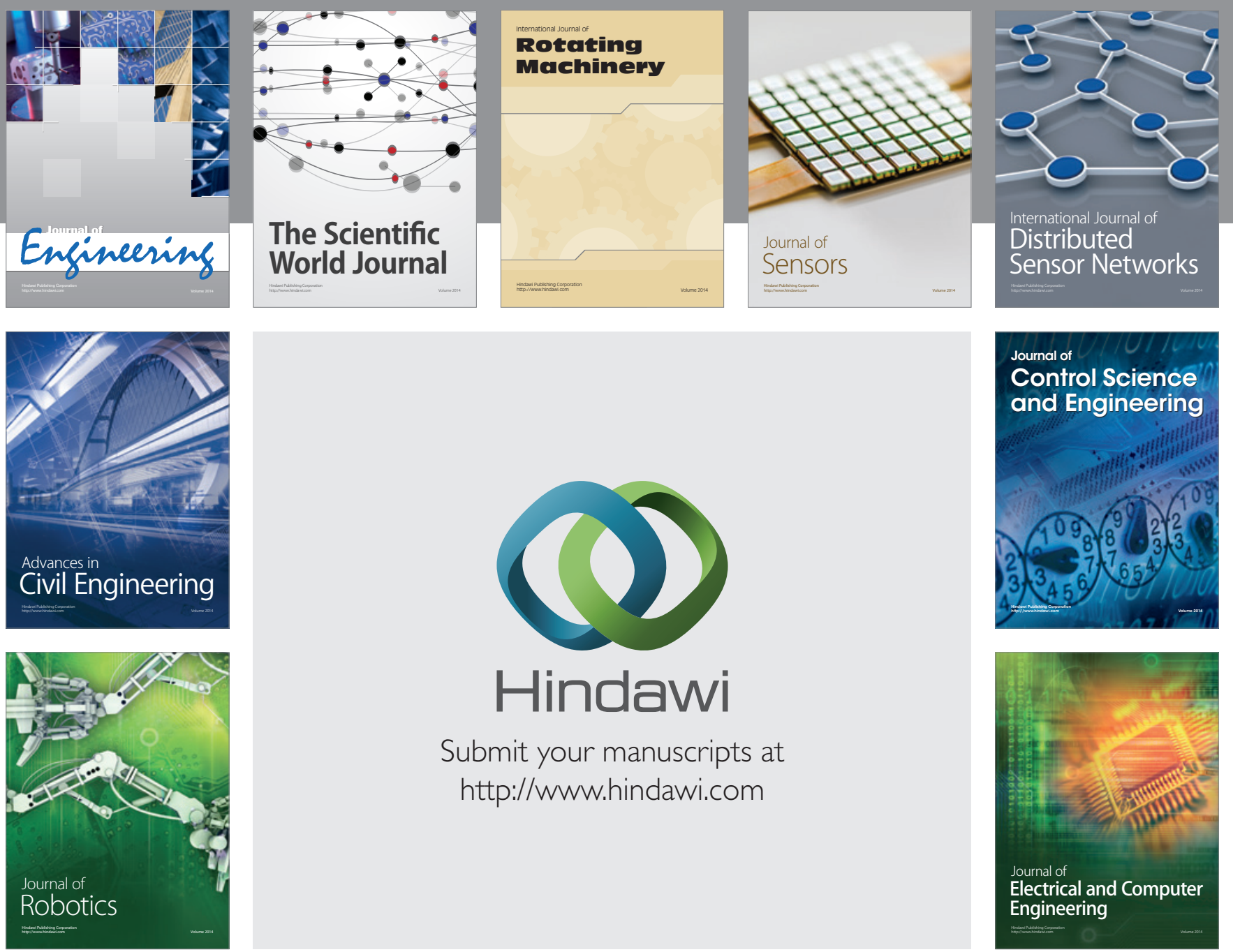

Submit your manuscripts at

http://www.hindawi.com
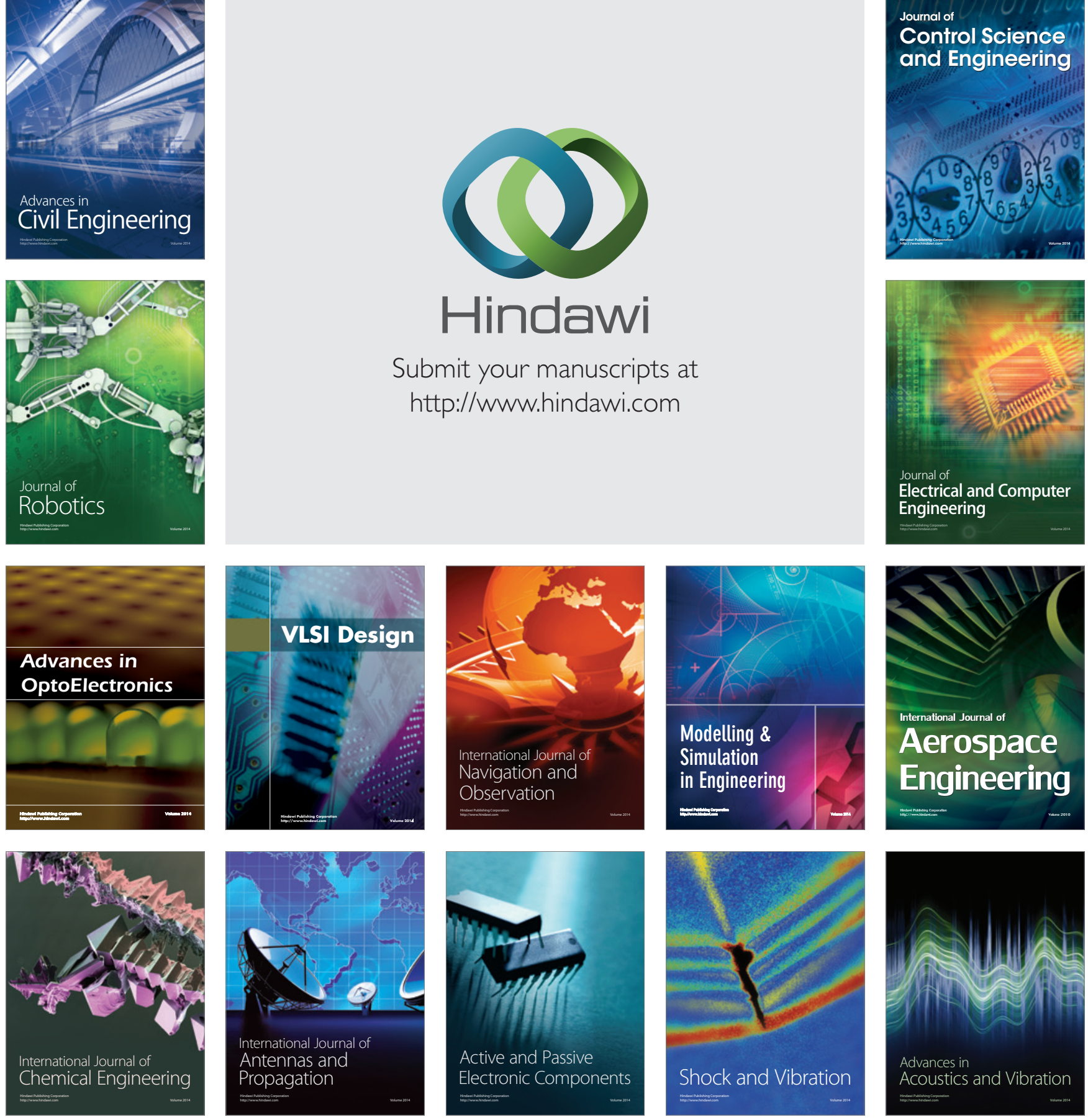